\title{
Black Hole Motion as a Trigger of Orbital Resonances
}

\author{
Christian M. Boily ${ }^{1}$, Nicolas Gaudin ${ }^{1}$, and Sabine Richard ${ }^{1}$ \\ ${ }^{1}$ Observatoire astronomique de Strasbourg \& CNRS UMR 7550 \\ 11, rue de l'université, Strasbourg F-67000, France \\ Email: christian.boily@astro.unistra.fr
}

\begin{abstract}
The motion of a black hole (BH) induces a strong response from the surrounding stellar population. We recall the case of an analytical harmonic potential where half of the stars on circular orbits in that potential shift to an orbit of lower energy, while the other half receive a positive boost and recede to a larger radius. The black hole itself remains on an orbit of fixed amplitude and merely acts as a catalyst for the evolution of the stellar energy distribution function $f(E)$. Numerical integration with a response code show that this effect is operative out to a radius of $\sim 3$ to 4 times the radius of influence of the $\mathrm{BH} R_{\mathrm{BH}}$. The results for the kinematic signature of $\mathrm{BH}$ motion are not sensitive to the shape of the potential and the temperature of the stars measured with Toomre's $Q$ parameter. We report on the evolution of fully self-gravitating systems when damping of the $\mathrm{BH}$ orbit is way off the expected exponential decay obtained from the Chandrasekhar formula, a possible result from energy exchanges with the background stars.
\end{abstract}

Keywords. galaxies: nuclei, black hole physics

The supermassive $\mathrm{BH}$ at the center of galaxies is often modeled as being at rest, owing to rapid orbital decay through dynamical friction (e.g., Tremaine et al. 1994). We have shown that this orbital decay may induce a long-lasting response in the kinematics of surrounding stars (Boily, Padmanabhan, \& Paiement 2008). A toy model helps us understand the origin of this slow decay: let us set a $\mathrm{BH}$ on a radial path of amplitude $R_{0}$ down the $x$-axis (say) in a background harmonic gravitational potential. The orbits have an angular frequency $\omega$ for both $\mathrm{BH}$ and a test star orbiting near the center, but their motion is out of phase by $\phi_{0} \in[0,2 \pi]$. The net force $F$ acting on the star is the sum of a radial component $F_{\mathrm{r}}$ and a force parallel to the $x$-axis, which we take to be of the form $F_{x} \cos \left(\omega t+\phi_{0}\right)$. The net mechanical work done by the $\mathrm{BH}$ over one period is the integral $\delta W=\int F \cdot v d t$. We find that $\delta W$ changes sign, but keeps the same amplitude, when the phase $\phi_{0}$ shifts to $\phi_{0}+\pi$. Thus, whenever the stellar phase-space density is well sampled, half the stars receive $(\delta W>0)$ mechanical energy from, and half give off $(\delta W<0)$ energy to, the BH. Expanding this calculation to 3-D indicates a stronger response from near-coplanar stellar orbits. We have recently carried out fully self-consistent $N$-body calculations of this applied to core and Hernquist (1990) potentials, and find that the orbital decay of the $\mathrm{BH}$ is much closer to exponential in a peaked density profile. A survey of $\gamma$-models will be published in due course.

\section{References}

Boily, C. M., Padmanabhan, T., \& Paiement, A. 2008, MNRAS, 383, 1619

Hernquist, L. 1990, ApJ, 356, 359

Tremaine, S. D., Richstone, D. O., Byun, Y.-I., Dressler, A., Faber, S. M., Grillmair, C., Kormendy, J., \& Lauer, T. R. 1994, AJ, 107, 634 